\title{
Reduced port laparoscopic surgery for colon cancer in a patient with tuberculous kyphosis and dwarfism: a rare case and literature review
}

\author{
Si-Yuan Yao, Atsushi Ikeda, Yoichiro Tada \\ Kobe City Medical Center West Hospital, Kobe, Japan
}

Videosurgery Miniinv 2015; 10 (2): 275-281 DOI: 10.5114/wiitm.2015.52558

\begin{abstract}
With accumulated surgical experience, the contraindications to laparoscopic surgery have been decreasing. Reduced port laparoscopic surgery has been widely adopted for a variety of diseases. However, surgery in patients with anatomic deformities are still a challenge for surgeons, specifically abdominal surgery in patients with severe kyphosis. A 71-year-old man with a diagnosis of ascending colon cancer had severe kyphosis with extremely short stature, secondary to tuberculous spondylitis. Laparoscopic right hemicolectomy was successfully performed with a single umbilical incision plus one port. This is the first reported case involving laparoscopic surgery in a patient with tuberculous kyphosis. The purpose of this report is to describe the surgical skills of reduced port laparoscopic surgery in a patient with altered habitus. With proper planning and a meticulous operation, minimally invasive surgery could be safely achieved.
\end{abstract}

Key words: reduced port laparoscopic surgery, tuberculous kyphosis, dwarfism.

\section{Introduction}

In recent years, reduced port laparoscopic surgery, including single incision laparoscopic surgery (SILS), has been widely adopted for a variety of diseases. The potential benefits of this approach include less postoperative incisional pain, less risk of incisional hernia, fewer wound complications, and improved cosmetic outcomes [1]. As laparoscopic experience accumulates in the surgical community, minimally invasive approaches should always be considered to achieve these benefits in patients. However, in patients with anatomic deformities, such as kyphosis or dwarfism, this approach is still challenging for surgeons, and there are few reports about the appropriate procedure.

The purpose of this report is to describe our rare experience in performing laparoscopic surgery in a patient with an altered habitus that was unique to tuberculous spondylitis (TS).

\section{Case report}

The patient was a 71-year-old man who was diagnosed with ascending colon cancer. Colonoscopy revealed an encircling mass in the ascending colon, which biopsy confirmed to be adenocarcinoma (Photo $1 \mathrm{~A})$. A contrast computed tomography (CT) scan showed enhanced wall thickening in the ascending colon with no distant metastasis (Photo $1 \mathrm{~B}$ ). The patient's medical history included childhood tuberculosis, and he had severe kyphosis secondary to TS. In the standing position, he presented a typical shape of TS: kyphosis with extreme short stature and an anteriorly tilting thoracic cage (Photos $2 \mathrm{~A}, \mathrm{~B}$ ).

\section{Address for correspondence}

Si-Yuan Yao MD, Kobe City Medical Center West Hospital, 2-4, Ichibancho, Nagata-ku, 653-0013 Kobe, Japan, phone: 819053763581 , 

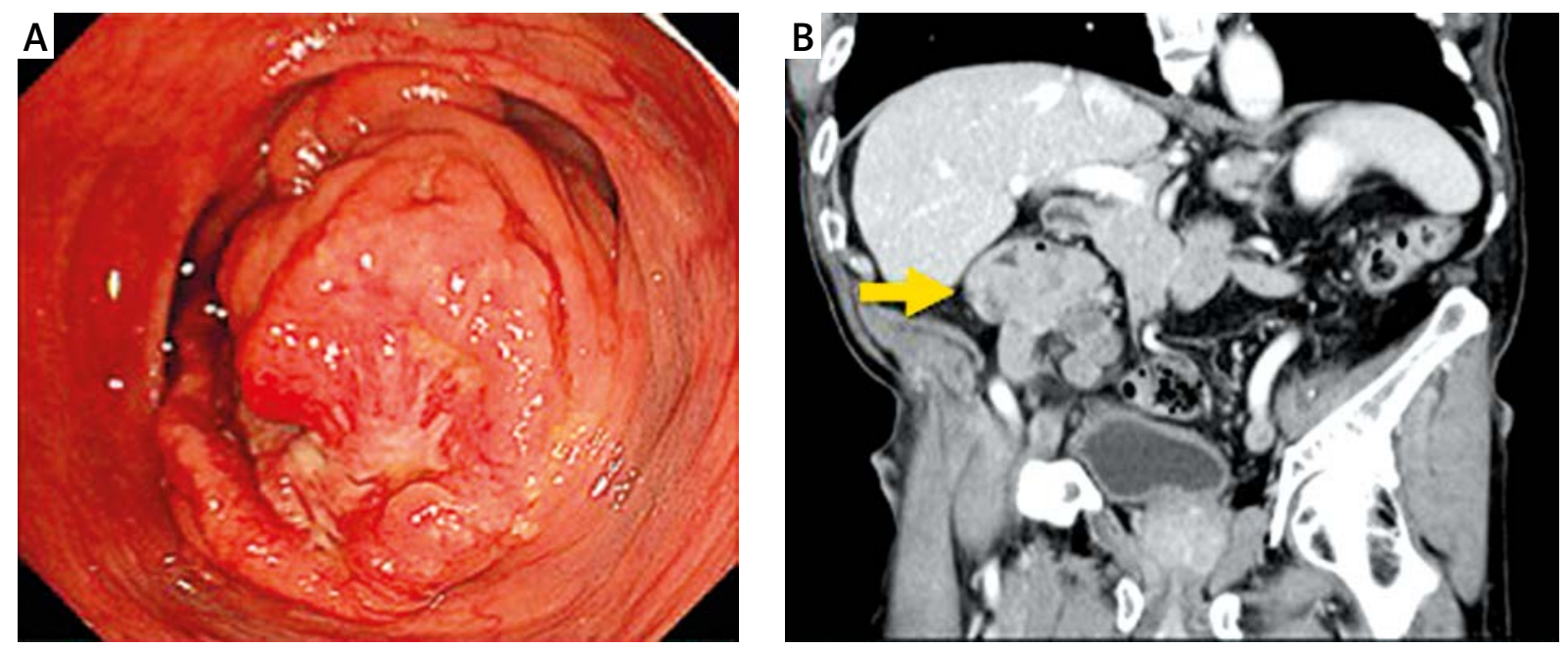

Photo 1. A - Colonoscopy revealed an encircling mass in the ascending colon. B - A contrast-enhanced computed tomography scan showed enhanced wall thickening in the ascending colon (arrow). A coronal plane scan also showed the shortened distance between the ribs and iliac crest

Pectus carinatum was also observed (Photo $2 \mathrm{C}$ ). The anteriorly tilting thoracic cage resulted in low-lying costal margins and a narrow abdomen. A CT of the dorsolumbar spine showed complete destruction of D10 to L5. There was marked kyphosis at the seat of the disease and compensatory lordosis of the thoracic vertebrae and sacral bone (Photo 2 D). Fortunately, the patient had no neurological deficits even with this $90^{\circ}$ deformity of the vertebrae and spinal cord. The CT angiography showed distorted anatomy of the abdominal aorta (Photo $2 \mathrm{E}$ ). The aorta was deformed by kyphotic growth of the affected vertebrae.

Though a curative operation for his cancer was planned, selecting an appropriate approach was difficult. Considering his narrow abdomen, laparoscopic surgery with a minimal number of trocar insertions was selected. The patient's height was $128 \mathrm{~cm}$, and his weight was $41 \mathrm{~kg}$. His body mass index was $25.0 \mathrm{~kg} / \mathrm{m}^{2}$. No restrictive or obstructive lung disease was found on pulmonary function tests.

General anesthesia was induced and the patient was placed in the supine position with legs spread apart. The thoracic cage presented as dome shaped owing to the compensatory lordosis of the dorsal vertebrae and pectus carinatum. The low-lying costal margins were only remarkable laterally. Though the direct distance between the xiphoid process and pubic tubercle was shortened to $17 \mathrm{~cm}$, there was a 10-cm gap in the height between them (Photo $3 \mathrm{~A}$ ). This dome-shaped thoracic cage with height was helpful for expanding the abdomen via pneumoperitoneum, providing a wide operative field (Photo $3 \mathrm{~B}$ ).

Laparoscopic right hemicolectomy with umbilical single incision plus one port was performed. First, a trans-umbilical 3-cm incision was made with the Hasson technique. A small wound retractor (Lap-Protector, Hakko, Nagano, Japan) and a single port device (EZ Access, Hakko, Nagano, Japan) were used. Two 5-mm trocars and a $10-\mathrm{mm} 30^{\circ}$ rigid endoscope were used. An additional 5-mm trocar was inserted in the right lower quadrant for assistant use (Photo $3 \mathrm{C}$ ). The surgeon stood between the patient's legs. On the patient's right side, the first assistant facilitated retraction. On the patient's left side, the second assistant handled the scope. After pneumoperitoneum was induced, an unexpectedly wide abdominal cavity was achieved. The carbon dioxide pneumoperitoneum pressure was maintained to $12 \mathrm{~mm} \mathrm{Hg}$ during the operation. The working space was not divided by the costal arches (Photo $3 \mathrm{D}$ ). Therefore, insertion of the trocar was relatively easy (Photo $3 \mathrm{E}$ ). The surgical technique was similar to conventional laparoscopic right hemicolectomy and involved a medial-to-lateral approach. We used a Harmonic Ace (Ethicon, Cincinnati, OH, USA) for dissection of lymph nodes and vessels. All lymph nodes and connective tissue around the ileocolic vessels and right branch of the midcolic vessels were successfully dissected. The vessels were divided using Endo clips (Covidien, Minneapolis, MN, USA). All soft tissue anterior to the superior mesenteric vein was completely removed and 

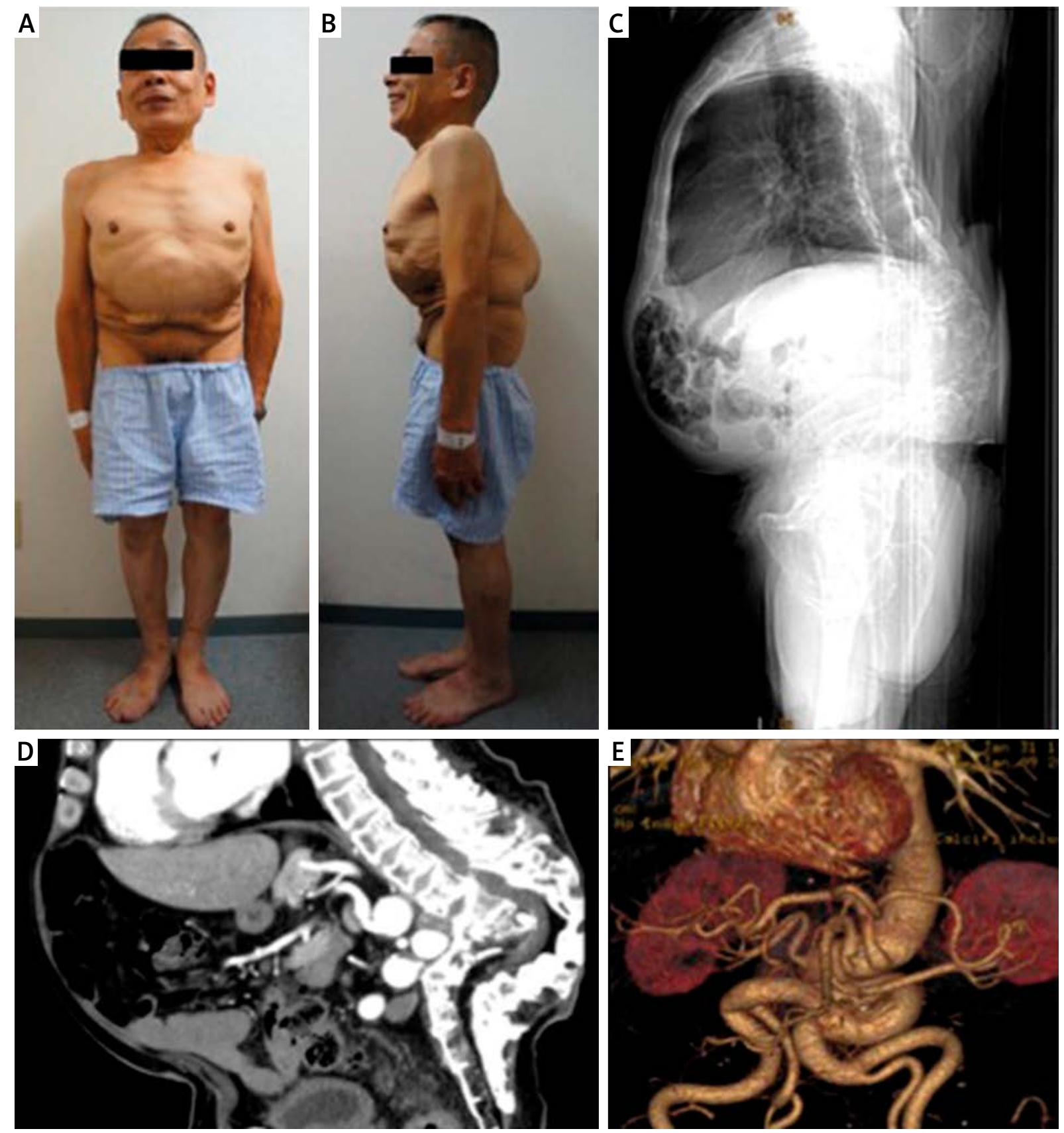

Photo 2. A, B - The patient in the standing position: kyphosis with extreme short stature and anteriorly tilting thoracic cage. C - Radiography in the sagittal plane showed pectus carinatum. D - D10 to L5 were completely destroyed with $90^{\circ}$ kyphotic malformation of the vertebrae and spinal cord. E - Distorted anatomy of abdominal aorta

D3 lymph node dissection was performed (Photo $3 \mathrm{~F}$ ). The specimen was retrieved through the umbilical port without wound extension. Extracorporeal functional end-to-end anastomosis was performed using Endo GIA (Covidien, Minneapolis, MN, USA). The operation was finished without drain placement. The operative time was 160 min with minimal blood loss $(<20 \mathrm{ml})$. The postoperative course was uneventful. The patient was discharged 9 days after surgery.

The removed tissue specimen was $25 \mathrm{~cm}$ in length with 10-cm surgical margins, and the tumor size was $5 \mathrm{~cm} \times 3 \mathrm{~cm}$ (Photo $4 \mathrm{~A}$ ). The pathologic 

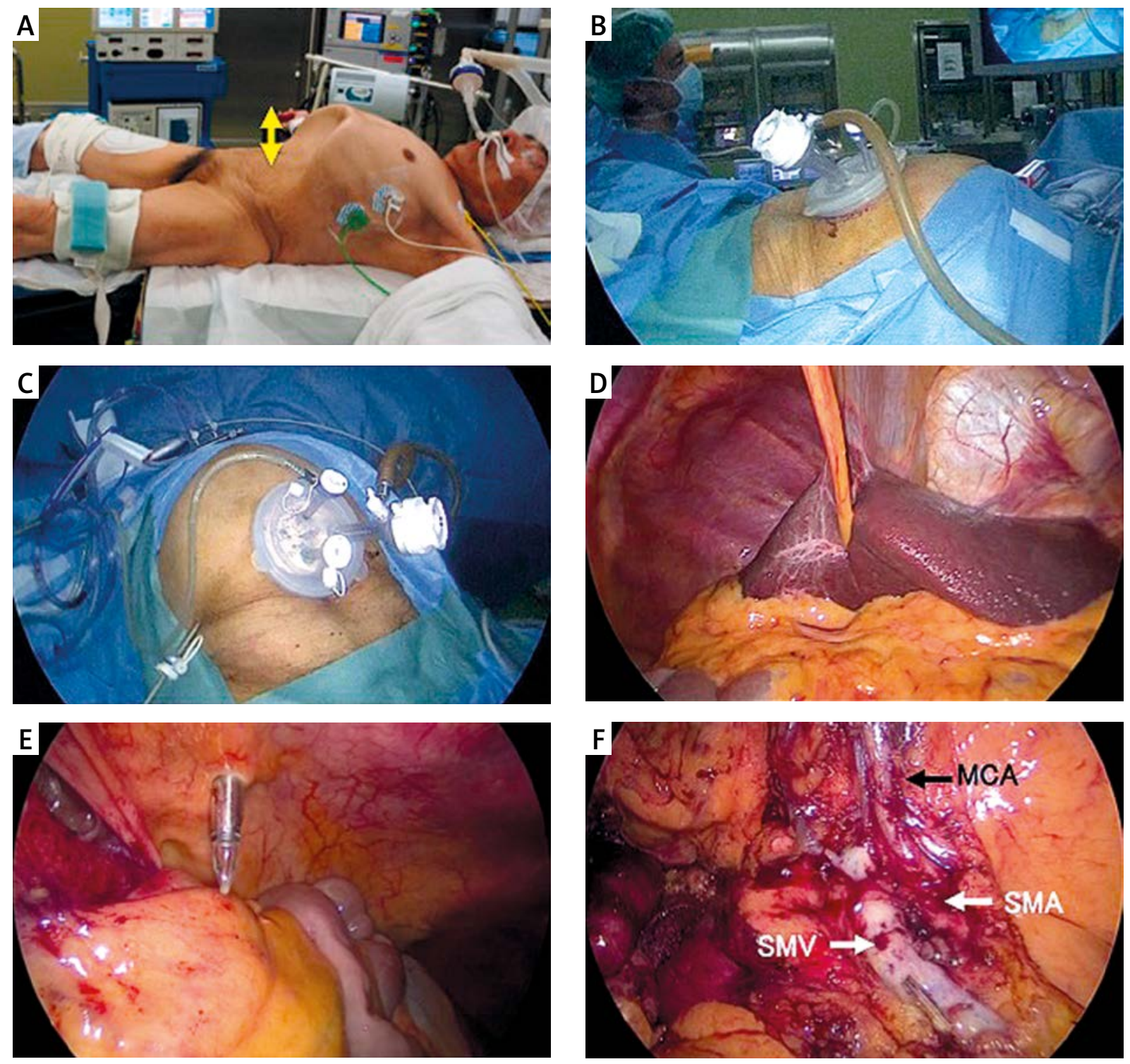

Photo 3. Findings during operation. A - Supine position on the operating table. There was a 10-cm gap in the height between the xiphoid process and the pubic tubercle (arrow). B - Extended abdominal wall after pneumoperitoneum. C - Port placement. Umbilical single incision and one additional port in the right lower quadrant. D - The operative field was not divided by the low-lying costal margin. E - Trocar insertion was performed without difficulty. F - Right hemicolectomy with D3 node dissection around the superior mesenteric vein (SMV) and superior mesenteric artery (SMA). Middle colic artery (MCA) is also indicated

staging of the tumor was T3N1M0, stage Illa. The patient was followed up with adjuvant chemotherapy. Scarring was almost invisible 3 months after the operation (Photo 4 B).

\section{Discussion}

Tuberculous spondylitis, also known as Pott disease, is one of the oldest demonstrated diseases in humans, having been documented in spinal remains from the Iron Age in Europe and in ancient mummies from Egypt [2]. While it is now rare in the developed world, it still exists in Asian and African countries. In Japan, according to the annual report of the Ministry of Health, Labour and Welfare, the 2013 morbidity rate of tuberculosis was 16.1 per 100,000, which was much higher than in other developed counties. Bone and joint involvement is observed in $1.5 \%$ of 

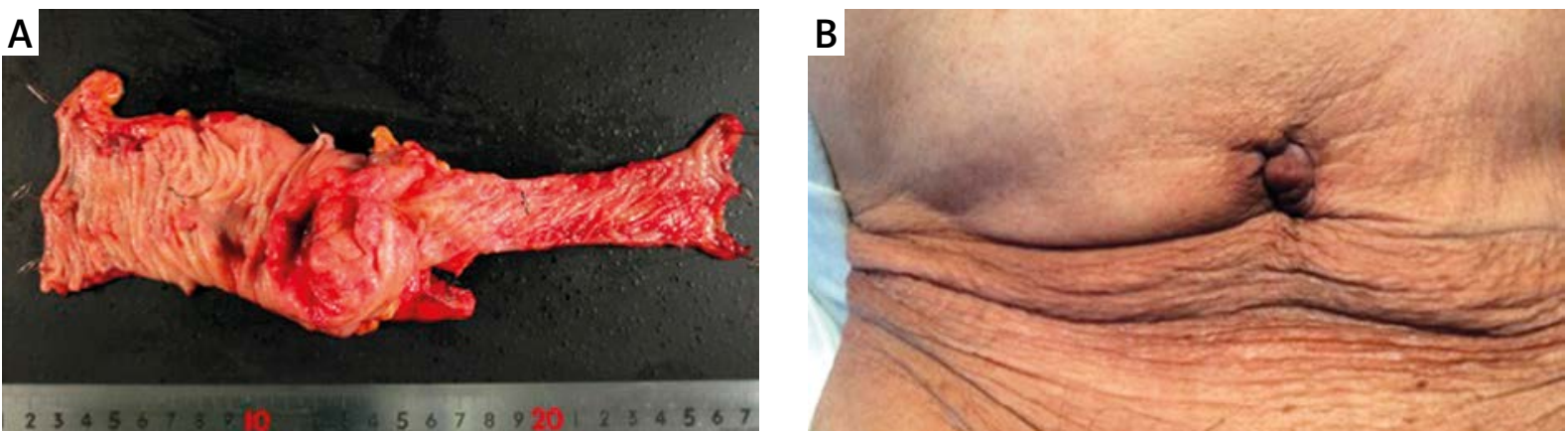

Photo 4. A - The resected specimen. B - The scarring 3 months after the operation

tuberculosis patients, with more than half of them having TS. Tuberculous spondylitis is caused by hematogenous spread of tuberculosis from other sites, often pulmonary, and characterized by caseous necrosis mainly in the lower thoracic and upper lumbar vertebrae. Because the anterior aspect of the vertebral body adjacent to the subchondral plate is usually affected, progressive bone destruction leads to vertebral collapse and kyphosis. In children, more severe destruction is seen than in adults, as most of the vertebral bodies are still cartilaginous. Even when a tuberculous lesion heals after anti-tuberculosis chemotherapy, kyphosis continues to progress with growth into adulthood. Therefore, a patient with childhood TS is often characterized by kyphosis and secondary dwarfism. When instability as a result of kyphotic deformity or neurological deficits emerges, surgical intervention is indicated [3].

Though this patient had no history of previous surgery, his body habitus was typical of childhood TS, with severe kyphotic deformity and short stature. One surgical concern when performing abdominal surgery in patients with severe kyphosis and dwarfism is selection of an appropriate surgical approach. The thoracic cage is often tilted anteriorly, resulting in low-lying costal margins and a narrow abdomen. A low-lying costal margin can divide the operative field [4]. Tajima et al. reported that a low-lying costal arch is a substantial risk factor for conversion to a laparotomy when performing a laparoscopic cholecystectomy [5]. The narrow abdomen can also cause difficulty with trocar insertion [6]. In contrast, open surgery results in difficulty obtaining a sufficient skin incision because of the shortened distance between the xiphoid process and pubic tubercle. A second surgical consideration in these patients is that abnormalities of the spine can alter the contour of the pelvic vessels. Kyphosis, scoliosis, and other skeletal abnormalities can affect the vascular anatomy and must be considered during trocar insertion and lymph node dissection [7].

In our case, the low-lying costal margin did not divide the operative field. Because of the compensatory lordosis above and below the affected vertebrae, the patient could lie supine. Pectus carinatum also made the thoracic cage larger, resulting in a wide working space after pneumoperitoneum was induced. In this point of view, tuberculous kyphosis is different from kyphosis in ankylosing spondylitis or osteoporosis. However, owing to the anteriorly tilting thoracic cage, the abdominal wall was too narrow to insert multiple trocars. Therefore, laparoscopic surgery with minimal trocar placement was selected. We chose not to perform SILS because of its own unique challenges, including the difficulty of achieving traction for triangulation formation, the inline vision, and the clashing of instruments. Some of these problems can be overcome with an additional trocar placement. For a patient with abnormal anatomy, tensionless dissection would result in unexpected vessel injury. To acquire appropriate traction, an additional trocar was inserted for the assistant's use. Further, Bae et al. reported that single plus one port laparoscopy might decrease collisions between laparoscopic instruments and the scope in a narrow bony operative field, while maintaining the cosmetic advantages of SILS [1]. Despite the distorted aorta, the superior mesenteric artery and superior mesenteric vein were found in their normal positions. To avoid vessel injury, preoperative CT angiography is essential. With proper planning and meticulous surgery, the minimally invasive approach of an umbilical single incision plus one port was safely achieved.

A literature search of the PubMed database between 1950 and 2015 retrieved 6 cases of laparoscopic surgery performed in patients with severe kyphosis (Table I) $[4,6,8,9]$. Osteoporosis was the most com- 


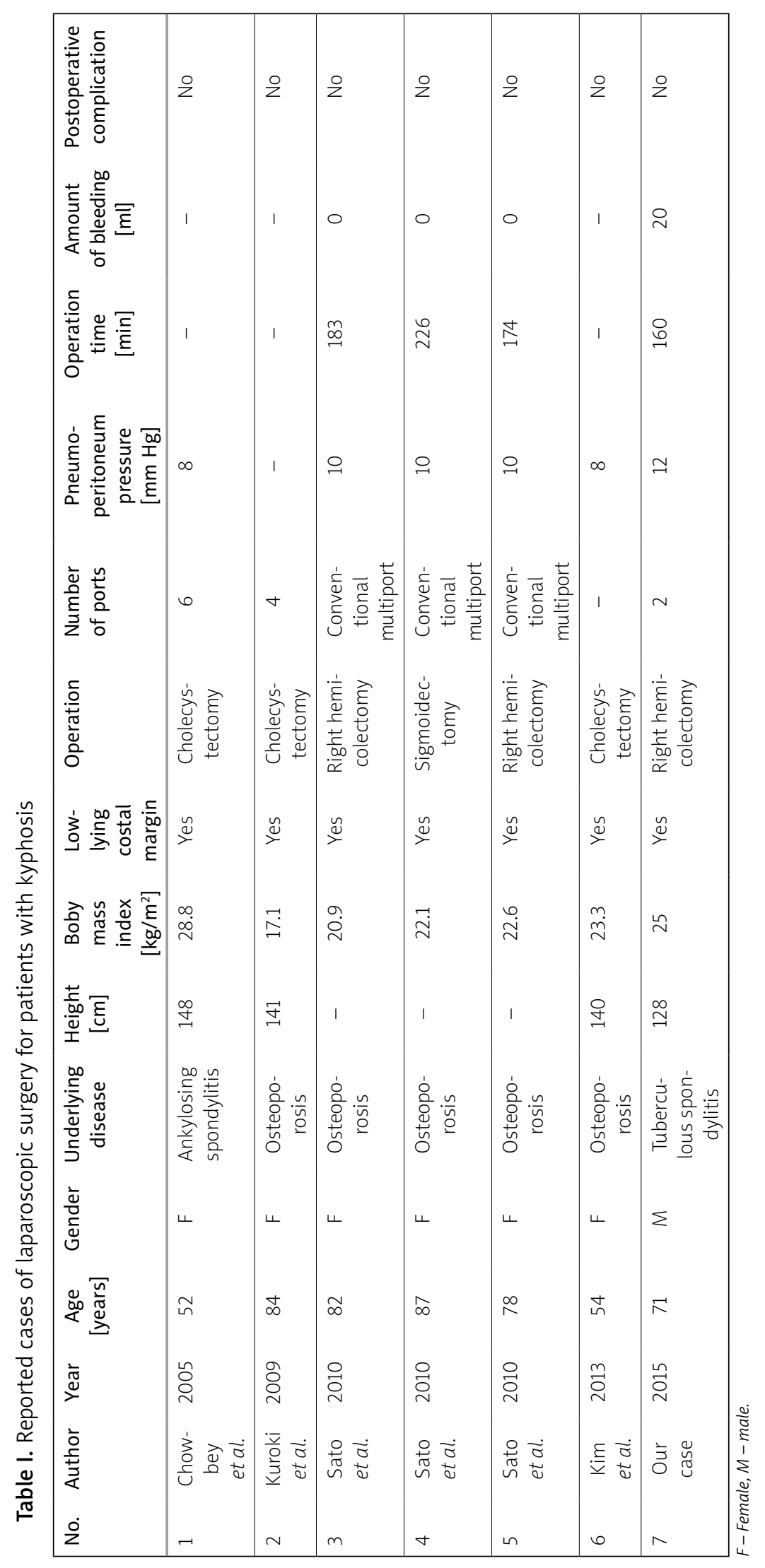


mon cause of kyphosis and ankylosing spondylitis was also responsible. All patients had low-lying costal margins. Sato et al. reported 3 cases of colectomy and the others were cholecystectomy. Though the number of trocars used was not mentioned in Sato et al.'s report, a conventional multiport approach seems to be what it describes. All cases, including ours, had uneventful postoperative courses. To the best of our knowledge, this is not only the first case report describing a laparoscopic surgery performed in a patient with tuberculous kyphosis but also the first report describing reduced port laparoscopic surgery performed in a patient with severe kyphosis and dwarfism.

\section{Conclusions}

As TS is not uncommon, surgeons are likely to encounter these patients in the clinical setting. Here, we have described our rare experience of abdominal surgery with minimally invasive technique for a patient with tuberculous kyphosis and dwarfism. Reduced port laparoscopic surgery appears to be a safe surgical option for patients with altered habitus or abnormal anatomy.

\section{Conflict of interest}

The authors declare no conflict of interest.

\section{References}

1. Bae SU, Baek SJ, Min BS, et al. Reduced-port laparoscopic surgery for a tumor-specific mesorectal excision in patients with colorectal cancer: initial experience with 20 consecutive cases. Ann Coloproctol 2015; 31: 16-22.

2. Taylor GM, Murphy E, Hopkins R, et al. First report of Mycobacterium bovis DNA in human remains from the Iron Age. Microbiology 2007; 153: 1243-9.

3. Rasouli MR, Mirkoohi M, Vaccaro AR, et al. Spinal tuberculosis: diagnosis and management. Asian Spine J 2012; 6: 294-308.

4. Chowbey PK, Panse R, Khullar R, et al. Laparoscopic cholecystectomy in a patient with ankylosing spondylitis with severe spinal deformity. Surg Laparosc Endosc Percutan Tech 2005; 15: 234-7.

5. Tajima Y, Kuroki T, Kitasato A, et al. Prediction and management of a low-lying costal arch which restricts the operative working space during laparoscopic cholecystectomy. J Hepatobiliary Pancreat Sci 2011; 18: 60-6.

6. Kim BS, Joo SH, Joh JH, et al. Laparoscopic cholecystectomy in patients with anesthetic problems. World I Gastroenterol 2013; 19: 4832-5.

7. Gordon LA, Shapiro SJ, Daykhovsky L. Problem-solving in laparoscopic surgery. Surg Endosc 1993; 7: 348-55.
8. Kuroki T, Tajima Y, Tsuneoka N, et al. Rib-lifting method for retraction of the low-lying costal arch in laparoscopic cholecystectomy of gallbladder torsion with kyphoscoliosis. Hepatogastroenterology 2009; 56: 1268-9.

9. Sato T, Yamaguchi S, Ozawa S, et al. Is laparoscopic surgery a contraindication in patients with severe senile kyphosis? Hepatogastroenterology 2010; 57: 1095-8.

Received: 9.04.2015, accepted: 25.05.2015. 